\title{
NON-HOMOGENEOUS MARKOV CHAINS WITH A FINITE STATE SPACE AND A DOEBLIN TYPE THEOREM
}

\section{RITA CHATTOPADHYAY}

Department of Mathematic:

Eastern Michigan University

Ypsilanti, MI 48197

(Received December 4, 1992 and in revised form March 4, 1994)

\begin{abstract}
Doeblin [1] considered some classes of finite state nonhomogeneous Markov chains and studied their asymptotic behavior. Later Cohn [2] considered another class of such Markov chains (not covered earlier) and obtained Doeblin type results. Though this paper does not present the "best possible" results, the method of proof will be of interest to the reader. It is elementary and based on Hajnal's results on products of nonnegative matrices.
\end{abstract}

KEY WORDS AND PHRASES. Stochastic matrices, convergence, state space, classes, period. 1991 AMS SUBJECT CLASSIFICATION CODE. 60J10.

\section{INTRODUCTION.}

Let $\left\{X_{n}: x \geq 0\right\}$ be a non-homogeneous Markov chain with finite state space $E=\{1,2, \cdot, S\}$ defined on some probability space $(\Omega, \Sigma, P)$. Let $\left(P_{n}\right)$ be the secuence of transition probability $(s$ by $s)$ matrices such that $\left(P_{n}\right)_{1 j}=$ the entry on the $\imath$ th row and $j$ th column of $P=P\left(X_{n+1}=\jmath \mid X_{n}=\imath\right),\left(P_{m, n}\right)_{\imath \jmath}=\left(P_{m+1} P_{m+2} \cdots P_{n}\right)_{\imath \jmath}=P\left(X_{n+1}=j \mid X_{m+1}=\imath\right), 0 \leq m<$ $n$. [It will be assumed that the matrices $P_{n}$ are all stochastic, i.e., every row sum is one; this means that when $P\left(X_{n}=\imath\right)=0$, the $\imath$ th row of $P_{n}$ can be defined in any way as long as it is nonnegative and has sum 1.] In [1], Doeblin considered classes of non-homogeneous Markov chains satisfying condition $(A): \exists$ a positive number $\delta \ni \forall(i, \jmath) \in E \times E$, either $\left(P_{n}\right)_{\mathfrak{\imath}}>\delta \forall n$ or $\left(P_{n}\right)_{\imath \jmath}=0 \forall n$. He also studied more general chains:

CONDITION (B). $\exists \mathrm{m} \delta>0$ and some positive integer $N \ni \forall(i, j) \in E \times E$, either $\left(P_{n}\right)_{\imath}>\delta$ for $n>N$ or $\lim \left(P_{n}\right)_{\imath \jmath}=0$ as $n \rightarrow \infty$.

Cohn [2] made a detailed study of Doeblin's paper [1] and these conditions in the context of Doeblin type results. Cohn [2] also studied chains satisfying conditions even more general than Doeblin's. The most general condition studied in Cohn's paper is:

CONDITION (B*). $\exists \delta>0 \ni \lim \max \left\{\left(P_{n}\right)_{\imath \jmath} \mid \imath, \jmath \ni\left(P_{n}\right)_{\imath \jmath}<\delta\right\}=0$ as $n \rightarrow \infty$.

The aim of this paper is to study non-homogeneous Markov chains satisfying conditions essentially different from the above conditions (where one does not require any kind of limit for the sequence $\left(P_{n}\right)_{\imath \jmath}$ or the sequence $\max \left(\left(P_{n}\right)_{\imath \jmath}:(\imath, j) \varepsilon A_{n}\right), A_{n}$ in $\left.E\right)$ in the context of Doeblin theory. For example, if one considers a non-homogeneous Markov chain where the transition matrices $\left(P_{n}\right)$ satisfy for some $(\imath, \jmath) \in E \times E$ the condition: $\left(P_{k(n)}\right)_{\imath \jmath}>\varepsilon_{k}>0, k(n)=k^{n}, n>0$, where $k$ is a prime integer and $\lim \varepsilon_{k}=0$ as $k \rightarrow \infty$, then this chain does not belong to the classes of chains studied in [2,3]. As one will see shortly, these chains (for $\varepsilon_{k}=1 / \log k$ )) are a type of chains that will satisfy the condition $(*)$ below that define the chains studied in this paper.

In this paper. Doeblin type results are obtained for non-homogeneous Markov chains satisfying the following condition:

CONDITION $(*)$. For any $(\imath, \jmath) \in E \times E$, either $\left(P_{n}\right)_{\imath \jmath}=0 \forall n$, or for $n$ sufficiently large, $\left(P_{n}\right)_{\imath} \geq 1 /(\log n)$. 
As will be clear from the proof, results of this paper actual holds under conditions more general than $(*)$. The present method of proof is different, and will be of interest to the reader.

\section{PRELIMINARIES.}

Throughout this and the next section, we will assume that the $P_{n}$ 's have the same skeleton, i.e., either $\left(P_{n}\right)_{\imath \jmath}=0 \forall n \geq 1$ or $\left(P_{n}\right)_{\imath \jmath}>0 \forall n \geq 1$. Define that $\imath \rightarrow j$ if $P\left(X_{n}=\jmath \mid X_{0}=\imath\right)>0$ for some $n \geq 1$. If $\imath \rightarrow \imath, \imath$ is self-communicating and define the period of $i, d(i)=$ g.c.d $\left\{n \mid\left(P_{k, k+n}\right)_{\mathfrak{a}}>0\right.$ for some $\left.k \geq 0\right\}$. In the parenthesis above the phrase "for some $k \geq 0$ " can be replaced by " $\forall k>0$ " without changing the definition since the $P_{n}$ 's have the same skeleton. Note that it is easily proven that the set $F=\{i \in E \mid i \rightarrow l\}$ is a nonempty subset of $E$ (since $E$ is finite). A state $\imath$, as usual, is called essential if $i \rightarrow j \Rightarrow \jmath \rightarrow \imath$. A state which is not essential is called unessential. All states in $E-F$ are unessential. As in the homogeneous case, $F$ is partitioned into equivalence classes with respect to the equivalence relation $\operatorname{irj}$ iff $\imath \rightarrow \jmath$ and $j \rightarrow \imath$. Then it is easily verified that all states within the same class have the scme period. Also, in class $G_{\alpha}$ with period $d_{\alpha}$ and any two states $i, j \in G_{\alpha}, \exists ! r_{\imath}, \ni 0 \leq r_{\imath}<d_{\alpha}$ and $\left(P_{m, n}\right)_{i}>0 \Rightarrow n-m=r_{\imath}(\bmod$ $d_{\alpha}$ ). [Recall: $P_{m, n}=P_{m+1} P_{m+2} \cdots P_{n}, m<n$ ]. Also, each class $G_{\alpha}$ with period $d_{\alpha}$ can be partitioned into sub-classes $C_{\jmath}, j=1,2, \cdots d_{\alpha}, \ni$ if $i \in C_{t 1}$ and $\jmath \in C_{t 2}$ then $\left(p_{m, n}\right)_{1},>0 \Rightarrow n-m=t_{2}-t_{1}\left(\bmod d_{\alpha}\right)$. [The proofs of the above assertions are the same as the homogeneous case in Chung's book [3]]. In the proof of our theorem, we need to apply Hajnal's weak ergodicity result in [4]. We explain what it is. A nonnegative square matrix is called allowable if $\exists$ at least one positive entry in each row and each column. For an allowable matrix $P$, Hajnal [4] defined $\Phi(P)$ as :

$$
\begin{aligned}
\Phi(P) & =\min \frac{P_{\imath k} P_{\jmath 1}}{P_{\jmath k} P_{\imath 1}}, \forall i, j, k, \ell \text { if } P \text { has all entries positive, } \\
& =0 \text {, otherwise }
\end{aligned}
$$

A sequence of sxs nonnegative matrices is called weakly ergodic if for each $m \geq 0$ and any $i, j, k$ in the state space $\frac{\left(P_{m, n}\right)_{\imath \jmath}}{\left(P_{m, n}\right)_{k \jmath}} \rightarrow V^{(m)}{ }_{z k}$ as $n \rightarrow \infty$, where the $V^{(m)}{ }_{i k}$ 's are independent of $j$. We need the following theorem: Theorem (Hajnal). A sequence of allowable matrices is weakly ergodic if $\exists$ a strictly increasing sequence of integers $\left(r_{m}\right) \ni \Sigma_{m=1} \sqrt{\Phi\left(P_{r m, r m+1}\right)}=\infty$.

\section{MAIN RESULTS.}

We now state the main theorem:

THEOREM 3.1. Let $\left(P_{n}\right)$ be a sequence of $s \times s$ stochastic matrices with state space $S$ such that they all have the same skeleton. Let us assume the following condition: "For each $i \in S$, let $E_{\mathrm{i}}=\{j \in S: i \leftrightarrow j\}$. Then for any two states $u, v \in E_{i}$, either $\left(P_{n}\right)_{u v}=0$ for all $n$ or for sufficiently large $n,\left(P_{n}\right)_{u v} \geq 1 /(\log n)$. "Then the following results hold: the state space $S$ can be partitioned as $S=T_{0} U\left(\bigcup_{\alpha} E_{\alpha}\right) U\left(U_{\beta} I_{\beta}\right)$, where $T_{0}$ contains all the non-self communicating elements, $E_{\alpha}$ 's the essential self-communicating classes and the $I_{\beta}$ 's the inessential self-communicating classes. Each $E_{\alpha}$ can further be partitioned into cyclical subclasses $E_{\alpha}=\bigcup_{u=1}^{d(\alpha)} E_{\alpha u}, d(\alpha)$ being the period of $E_{\alpha}$. Similarly, each $I_{\beta}$ can be partitioned as $I_{\beta}=\bigcup_{v=1}^{\alpha(\beta)} I_{\beta v}$, where $d(\beta)$ is the period of $I_{\beta}$. Also

(i) $\quad \lim \left(P_{m, n}\right)_{i j}=$ for $m \geq 0$ for all $i$, if $j$ in $T_{0}$ as $n \rightarrow \infty$

(ii) $\quad\left(P_{m, n}\right)_{\imath \jmath}=0$ for $m<n$ if $i$ in $E_{\alpha}$ and $j$ not in $E_{\alpha}$.

(iii) $\left(P_{m, m}\right)_{i j}=0$ of $n-m \neq v-u(\bmod d(\alpha))$, whenever $j$ in $E_{\alpha u}, i$ in $E_{\alpha v}$. A similar result holds when $i$ in $I_{\beta v}$ and $j$ in $I_{\beta u}$.

(iv) If $i \in E_{\alpha u}, j \in E_{\alpha v}$, and $n-m=v-u(\bmod d(\alpha))$, then $\left(P_{m n}\right)_{\imath \jmath}=\left(P_{n}\right)_{\jmath}+\left(\varepsilon_{m, n}\right)_{\imath \jmath}$, where $\lim \left(\varepsilon_{m, n}\right)_{\imath \jmath}=0$ and $\lim \Sigma_{\jmath \varepsilon E_{\alpha v}}\left(P_{n}\right)_{\imath \jmath}=1$ as $n \rightarrow \infty$. 
(v) If $\iota, k \in I_{\beta u}$ and $\jmath \in I_{\beta v}, n-m=v-u(\bmod d(\beta))$, then $\operatorname{llm}\left[\left(P_{m, n}\right)_{\imath \jmath} /\left(P_{m, n}\right)_{k \jmath}\right]=v^{(m)}{ }_{i k}$ as $n \rightarrow \infty$.

(vi) Let $\jmath \in E_{\alpha u}, 1 \leq u \leq d(\alpha)$. Then for $\imath \in S,\left(P_{m, n}\right)_{\imath \jmath}=\left(P_{n}\right)_{\jmath} . \quad \Sigma_{k \varepsilon} E_{\alpha u}\left(P_{m, n}\right)_{\imath k}+\left(\varepsilon_{m, n}\right)_{t k}$ $+\left(\varepsilon_{m, n}\right)_{\imath}$, and $\lim \left(\varepsilon_{m, n}\right)_{\mathfrak{i}}=0$ as $n \rightarrow \infty$.

The idea of the proof is the following. First, to find a useful estmate of the integer $N$ (and this is one of the crucial steps in my proof) with the following property: $\left(P_{k, k+n d}\right)_{n}>0$ whenever $n \geq N$ where $d$ is the period of the element $\imath, \imath \notin T_{0}$. (The estimate is in terms of $d$ and the number $a$ which is the number of elements in the class containing 2 ). The second step is to consider restrictions of the sequence of blocks (each block is a product of length $d(\alpha)$ ) $P_{m, m+d(\alpha)}$ to an essential class (with period $d(\alpha)$ ); these restrictions are allowable nonnegative matrices and then use Hajnal's theorem to this sequence after estimating the $\Phi$ function (given in Hanjal's theorem) based on the estimate that I have obtained in the first step. The third step is consider a similar procedure for the unessential classes.

PROOF. We discuss the proof in several parts.

(1) Let $a, b$ be positive integers and $0<a<b$, g.c.d $\{a, b\}=(a, b)=d$. Then there exist integers $u$ and $v$ such that $u a+v b=d$ and $|v| \leq u \leq b$.

PROOF of (1). With no loss of generality, we can assume that $d=1$. It is known that there are integers $s$ and $t$ such that

$$
s a+t b=1 .
$$

Let $x$ be the greatest integer less than or equal to $\frac{b-s}{b}$. We claim that

$$
|t-a x| \leq s+b x \leq b .
$$

Notice that (3.2), once established, will complete the proof of (1), for

$$
(s+b x) a+(t-a x) b=1 .
$$

To establish (3.2), note first that

$$
\frac{-s-t}{b-a} \leq \frac{-s}{b} \leq \frac{t-s}{a+b} \leq \frac{b-s}{b}
$$

Write, $|s|=b q+r, 0 \leq r<b$, where $q$ and $r$ are integers. Let $s>0$. Then

$$
\begin{aligned}
\frac{b-s}{b}=1-q-\frac{r}{b} \text { so that } \frac{b-s}{b}-x & \leq 1-\frac{r}{b} \leq 1-\frac{1}{b(a+b)}=\frac{b-s}{b}-\frac{t-s}{a+b} . \Rightarrow \\
\frac{t-s}{a+b} & \leq x \leq \frac{b-s}{b} .
\end{aligned}
$$

If $\quad s<0 . \quad$ then $\quad \frac{b-s}{b}=1+q+\frac{r}{b} \Rightarrow \frac{b-s}{b}-x=\frac{r}{b} \leq 1-\frac{1}{b(a+b)}$. $r(a+b)<b(a+b) \Rightarrow r(a+b) \leq-1+b(a+b))$. This means (3.5) holds. Note that (3.5) implies that

$$
t-a x \leq s+b x \leq b
$$

Also, (3.4) implies that $\frac{-s-t}{b-a} \leq x$ or

$$
a x-t \leq s+b x .
$$

This establishes (3.2) and (1) is proven.

(2) Let $d=$ g.c.d. $\left\{n_{1}, n_{2}, \cdots, n_{k}\right\}$, where $1 \leq n_{1}<n_{2}<\cdots<n_{k}$ are positive integers. Then $\exists$ positive integers $c_{1}, c_{2}, \cdots, c_{k}$ such that

(i) $c_{1} \geq c_{2} \geq \cdots \geq c_{k}$

(ii) $\quad c_{1} n_{1}-c_{2} n_{2}-\cdots-c_{k} n_{k}=d$ 
(iii) If $d_{\imath}=$ g.c.d. $\left\{n_{1}, n_{2}, \cdots, n_{\imath}\right\}, 1 \leq \imath \leq k$, then $c_{k} \leq \frac{n_{k}}{d_{k}}, c_{k-1} \leq \frac{n_{k} n_{k-1}}{d_{k} d_{k-1}}$ etc. $c_{\mathrm{i}} \leq \frac{n_{\mathrm{a}} n_{\mathrm{a}}+1 \cdots n_{k}}{d_{\mathrm{i}} d_{\mathrm{i}}+1 \cdots d_{k}}$

PROOF OF (2). The proof follows easily using induction on $k$ and (1).

(3) Let $d$ be the period of a self-communicating class $F$ and let $a$ be the number of elements in this class. For a state $i$ in this class, define the set $A(i)=\left\{n \in z^{+}:\left(P_{k, k+n}\right)_{\mathbf{n}}>0\right.$ for all $K\}$. Also, let $A(a)=\left\{n \in z^{+}: n \leq a\right.$ and $n \in A(j)$ for $\jmath$ in $\left.F\right\}$. Then, $d=g . c . d . A(a)$.

PROOF OF (3). Notice that $d=$ g.c.d. $A(j)$ for each $j \in F$. Hence, $d \mid d_{0}$, where $d_{0}=$ g.c.d. $A(a)$. Now, let $n \in A(\imath)$. Then, $\left(P_{k, k+n}\right)_{\mathfrak{u}}>0$. If $n \leq a$, then $n \in A(a)$ and $d_{0} \mid n$. Let $n>a$. Since $i$ cannot lead to a state $\jmath$ outside $F$ (the class containing $i$ ), which can then lead to a state in $F$, it is clear that one can write $n=n_{1}+n_{2}+\cdots+n_{1}$, where each $n_{t}, 1 \leq t \leq \underline{1}$, is in $A(a)$. To see this, let $i=j_{n}$; then notice that $\left(P_{k, k+n}\right)_{\mathfrak{u}}=\Sigma\left(P_{k+1}\right)_{\imath \jmath_{1}}\left(P_{k+2}\right)_{\jmath_{1} \jmath_{2}} \cdots$ $\left(P_{k+n}\right) j_{n-1} j_{n}$. If $m$ is the smallest integer such that $j_{m}$ appears at least twice and $j_{m}=j_{m+p}$, then $a \geq p$ and $n=p+(n-p)$, where $\left(P_{k+m, k+m+p}\right)_{J_{m^{j}}}>0$ and $\left(P_{k, n-p+k}\right)_{\mathfrak{u}}>0$. This process is repeated. So $d_{0} \mid n$ since $d_{0} \mid n_{t}, 1 \leq t \leq \underline{1}$.

(4) Let $d$ be the period of a self-communicating class with $a$ elements and $N=\left\{\left[\frac{a}{d}\right] a\right\}^{2}$. $\forall n \geq N,\left(P_{k, k+n d}\right)_{u}>0, \forall k$ and states $i$ in this class.

PROOF of (4). Let $i$ be a state in this class. First, consider the shortest path from $i$ to $i$ through all the other states in this class which can be described as follows: $j_{0}=\underbrace{i \text { to } j_{1} \text { to } j_{2}}$ to etc.

$s_{1}$-steps $:_{2}$-steps

$$
\cdots \underbrace{j_{v} \text { to } i}_{s_{v+1} \text {-steps }}=j_{0}
$$

where all the $j_{1}$ 's are distinct and each $s_{1} \leq a$. If the length of this shortest path is $b$, then $d \mid b$ and $b \leq a^{2}$. Note that the corresponding shortest path for any other state $j$ in this class has also length $b$, since, for example, if $j=j_{1}$, then:

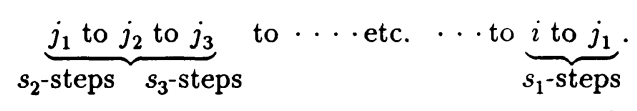

This information will be used later. Now, by step (3) $d=$ g.c.d. $\left\{n_{1}, n_{2}, \cdots, n_{t}\right\}, n_{\underline{1}}$ 's being distinct, each $n_{1} \leq a$ and for each $n_{1}, \underline{1} \leq 1 \leq t$, there is some state $i$ in the given class (equivalent) $\ni\left(P_{k}, k+n_{1}\right)_{12}>0$. By part (2), $\exists$ positive integers $c_{1} \geq c_{2} \geq \cdots \geq c_{t} \ni d=c_{1} n_{1}$ $-c_{2} n_{2}-\cdots-c_{t} n_{t}$. Let $\quad N_{0} d=c_{1} n_{2}+\cdots+c_{t} n_{t}$. Let $n \geq N_{0}\left(N_{0}-1\right)$. Then $n=a_{1} N_{0}\left(N_{0}-1\right)+a_{2} N_{0}+a_{3}$ where $a_{1} \geq 1, a_{2} \geq 0,0 \leq a_{3}<N_{0}$. Thus,

$$
\begin{gathered}
n d=a_{1}\left(N_{0}-1\right) \sum_{\underline{1}=2}^{t} c_{1} n_{1}+a_{2} \sum_{\underline{1}=2}^{t} c_{1} n_{1}+a_{3}\left(c_{1} n_{1}-\sum_{\underline{1}=2}^{t} c_{1} n_{1}\right) \\
=\sum_{\underline{1}=2}^{t}\left\{a_{1}\left(N_{0}-1\right)+a_{2}-a_{3}\right\} c_{\underline{1}} n_{1}+a_{3} c_{1} n_{1} .
\end{gathered}
$$

Note that by part (2),

$$
\begin{gathered}
c_{1} \leq \frac{n_{1}}{d} \cdot \frac{n_{2}}{d} \cdots \cdots \frac{n_{t}}{d} \leq \frac{2 d .3 d \ldots\left[\frac{a}{d}\right] d}{d^{t}} \leq\left[\frac{a}{d}\right] \\
\ni N_{0} d\left(N_{0}-1\right)=\left(c_{1} n_{1}-d\right)\left(c_{1} n_{1}-2 d\right) \frac{1}{d} \leq\left(\left[\frac{a}{d}\right] ! a-d\right)^{2} \cdot \frac{1}{d} .
\end{gathered}
$$

Note that if $m d=\sum_{\underline{1}=1}^{t} c_{1}{ }^{(m)} n_{\underline{1}}, c_{\underline{1}}^{(m)} \geq 0$, then $\left(P_{k, k+m d+b}\right)_{\mathfrak{u}}>0 \forall$ states $i$ in the class. [The reason is the following: Considering the shortest path of length $b$ from $i$ to $i$ through all the states in the class $i$ to $j_{1}$ to $j_{2}$ to $\cdots$ etc $\cdots$ to $j_{v}$ to $i$. Attach to this path an extra m.d steps in 
the most obvious manner, i.e., for each $j_{\underline{1}}$, there is an $n_{\underline{1}}$ such that $\left(P_{k_{2}, k+c_{\underline{1}}}(m)_{n_{\underline{1}}}\right)_{j_{\underline{1}} j_{\underline{1}}}>0$, so that the new path looks like

$$
i \text { to } \underbrace{j_{1} \text { to } j_{1}}_{c_{1}{ }^{(m)}{ }_{n_{1}} \text {-steps }} \text { to } \underbrace{j_{2} \text { to } j_{2}}_{c_{2}{ }_{2}-\text { - } \text { teps }} \text { to } \cdots \text { to } j_{v} \text { to } i] \text {. }
$$

Since, $b \leq a^{2}$ and $d \mid b, N_{0}\left(N_{0}-1\right)+\frac{b}{d} \leq \frac{1}{d^{2}}\left(\left[\frac{a}{d}\right]^{\prime} a-d\right)^{2}+\frac{b}{d} \leq\left(\left[\frac{a}{d}\right]^{l} a\right)^{2}$.

Therefore, if $n \geq\left(\left[\frac{a}{d}\right]^{\prime} \cdot a\right)^{2}$, then $n . d=m . d+b, m \geq N_{0}\left(N_{0}-1\right), \forall \quad \imath \quad$ in the class, $\left(P_{k, k+n d}\right)_{\mathfrak{u}}>0$.

(5) Let $G_{0}=\{\jmath \in S\}: \operatorname{llm}\left(P_{m, n}\right)_{\imath \jmath}=0, \forall m \geq 0, \forall$ states $i$ as $\left.n \rightarrow \infty\right\}$.

Since $S$ is finite, $G_{0}^{c} \neq \Phi$. Let $k \in G_{0}^{c} . \quad \exists \imath_{1} \in S$, $\ni \operatorname{llm}\left(P_{\left.m, n_{t}\right)_{1} k}>0\right.$ for some $m$ as $t \rightarrow \infty$. Or $g_{i_{1} k}^{(m)}>0$. Since

$$
g^{(m)} \imath_{1} k=\sum_{r=m+1}^{\infty} f^{m, r} \imath_{1, k} g^{\left({ }^{\prime}\right)} \exists r>m \in g_{k k}^{(r)}>0 .
$$

Thus $k$ is recurrent and $k \rightarrow k$.

(6) Let $T_{0}=\{j$ in $s: j \nrightarrow j\}$. Then $T_{0}$ in $G_{0}$. The set $T_{0}^{c}$ can be partitioned into equivalence classes with respect to the equivalence relation “ $\leftrightarrow$ ”. The equivalence classes in $T_{0}^{c}$ has either all essential or all unessential states.

(7) Let $\left\{E_{1}, E_{2}, \cdot, E_{e}\right\}$ be all the equivalence classes of $T_{0}^{c}$ consisting of only essential states. Each $E_{\alpha}$ can also be partitioned into subclasses $\left\{E_{\alpha 1}, E_{\alpha 2}, \cdots, E_{\alpha d(\alpha)}\right\}$, where $d(\alpha)$ is the period of the class $E_{\alpha}$ as follows: For a fixed $i$ in $E_{\alpha}, E_{\alpha r}=\left\{j \varepsilon E_{\alpha}:\left(P_{m, m+n}\right)_{\imath}>0\right) \Rightarrow n$ $=r(\bmod d(\alpha)))\}, 1 \leq r \leq d(\alpha)$. Clearly, for $j$ in $E_{\alpha \underline{1}}, k$ in $E_{\alpha \underline{1^{\prime}}},\left(P_{m, m+n}\right)_{\jmath k}>0$ implies that $n=\underline{1}^{\prime}-\underline{1}(\bmod d(\alpha))$. Note that the restriction of $P_{m, m+d(\alpha)}$ to $E_{\alpha d(\alpha)}$, i.e., $P_{m, m+d(\alpha)} \mid E_{\alpha d(\alpha)}$ is an allowable non-negative matrix because $\left(P_{m, m+d(\alpha)}\right)_{1 j}=0 \forall j$ in $E_{\alpha d(\alpha)}$, for some $i \in E_{\alpha d(\alpha)}$ and for some $m$, so that $\left(P_{m, m+d(\alpha)}\right)_{\mathfrak{u}}=0 \forall n$, which is a contradiction. Similarly, no column of $P_{m, m+d(\alpha) \mid E \alpha d(\alpha)}$ can be a zero column. For $i, j \in E_{\alpha d(\alpha)}, \exists$ a positive integer $k_{\imath} \leq\left[\frac{a}{d(\alpha)}\right] \ni \forall m$, $\left(P_{m, m+k_{i j} d(\alpha)}\right)_{1 j}>0$. This means that $n \geq N$ (where $N$ is as in $\left.(5)\right) \Rightarrow\left(P_{m, m+n d(\alpha)+k_{i j} d(\alpha)}\right)_{\mathfrak{y}}>0$. Let $M=\max \left\{N+k_{i j} ; i, j\right.$ in $\left.E_{\alpha d(\alpha)}\right\}$. Then $M \leq N+\left[\frac{a}{d(\alpha)}\right]$. By the assumption in the theorem, when $\left(P_{n}\right)_{\imath}>0$ for $i, j \in E_{\alpha}$, and $n$ sufficiently large,

$$
(*)\left(P_{n}\right)_{\imath \jmath} \geq \frac{1}{n^{\theta(a, d(\alpha))}}, \theta(a, d(\alpha))=\frac{1}{\left(N+\left[\frac{a}{d(\alpha)}\right]\right) d(\alpha)} .
$$

\{Notice that for $\left.i, j \in E_{\alpha d(\alpha)}\right\} \quad\left(P_{m, m+M d(\alpha)}\right)_{\imath \jmath}=\left(P_{m,\left[N+\left(M-N-k_{\imath \jmath}\right)\right] d(\alpha)+K_{\imath \jmath} d(\alpha)}\right)_{\mathfrak{\imath} \jmath}>0$, and $\forall k \geq 1$ and $n$ sufficiently large, by condition $(*)$, we have

$$
\left(P_{n+k M d(\alpha), n+(k+1) M d(\alpha)}\right)_{\mathfrak{z}} \geq \frac{1}{n+(k+1) M d(\alpha)} .
$$

It is clear that for $n$ sufficiently large $\Phi\left(\left.P_{n, n+M d(\alpha)}\right|_{E_{\alpha d(\alpha)}}\right) \geq \frac{1}{(n+M d(\alpha))^{2}}$.

Also, $P_{m, m+M d(\alpha)}\left|E_{\alpha d(\alpha)}=P_{n, n+M d(\alpha)}\right| E_{\alpha d(\alpha)} \cdots P_{m+(n-1) M d(\alpha), m+n M d(\alpha)} \mid E_{\alpha d(\alpha)}$. Thus, using Hajnal's theorem observe that the chain $P_{m, m+n M d(\alpha)} \mid E_{\alpha d(\alpha)}$ is weakly ergodic. That is the chain $P_{m, m+n d(\alpha)} \mid E_{\alpha d(\alpha)}$ is also weakly ergodic, because for $n>>n^{\prime}, P_{m, m+n d(\alpha)} \mid E_{\alpha d(\alpha)}$ $=P_{m, m+n^{\prime} d(\alpha) \cdot M \mid E_{\alpha d(\alpha)}}$ times. Due to weak ergodicity, $\left.i, j \in E_{\alpha d(\alpha)}, \mid\left(P_{m, n}\right)_{\imath \jmath}-\left(P_{m, n}\right)_{\jmath \jmath}\right) \mid \rightarrow 0$ as $n \rightarrow \infty$. If $n=r(n)(\bmod d(\alpha)), 0 \leq r(n)<d(\alpha)$, then for $m \geq d(\alpha) \ni n=m(\bmod d(\alpha))\left(P_{r(n), n}\right)_{\jmath \jmath}$ $=\sum_{1} \sum_{\text {in } E_{\alpha d(\alpha)}}\left(P_{r(n), m}\right)_{\jmath 1}\left(P_{m, n}\right)_{1}, \ni$ for $n=m(\bmod d(\alpha))$ and as $n \rightarrow \infty\left|\left(P_{r(n), n}\right)_{\jmath \jmath}-\left(P_{m, n}\right)_{\jmath \jmath}\right|$

$\rightarrow 0$. For $i, j \in E_{\alpha d(\alpha)}$ and $n=m(\bmod d(\alpha))\left(P_{m, n}\right)_{\mathfrak{\imath}}=\left(P_{r(n), n}\right)_{\jmath \jmath}+\left(\varepsilon_{m, n}\right)_{\mathfrak{\imath}}$, where $\lim \left(\varepsilon_{m, n}\right)_{\mathfrak{\jmath}}=0$ as $n \rightarrow \infty$. Writing $\left(P_{n}^{\prime}\right)_{\jmath}=\left(P_{r(n), n}\right)_{\jmath \jmath}$ then $\lim _{\jmath \in E_{\alpha d(\alpha)}}\left(P_{n}\right)_{\jmath}=1$ as $n \rightarrow \infty$. Let $i \in E_{\alpha \underline{\underline{1}}}, j \in E_{\alpha \underline{\underline{1}}}$,

$m<n, \ni n-m=\underline{1}-\underline{1}(\bmod d(\alpha)), 1 \leq \underline{1} \leq \underline{1}^{\prime} \leq d(\alpha)$. Then 


$$
\begin{aligned}
& \left(P_{m, n}\right)_{\imath \jmath}=\sum_{s \varepsilon E_{a \underline{1} \underline{1}^{\prime}}}\left(P_{m, m+\underline{1}^{\prime}-\underline{1}}\right)_{\imath s}\left(P_{m+\underline{1}-\underline{1}_{n}}\right)_{s \jmath} \\
& =\sum_{s \varepsilon E_{\alpha \underline{1}}}\left(P_{m, m+1^{\prime}-1_{i s}}\right)_{s}\left[\left(P_{n}\right)_{\jmath}+\left(\varepsilon_{n+\underline{1}^{\prime}-1, n^{\prime}}\right)_{s \jmath}\right]=\left(P_{n}\right)_{\jmath}+\left(\varepsilon_{m, n}^{\prime}\right)_{\imath \jmath}
\end{aligned}
$$

where $\quad\left(\varepsilon_{m, n}^{\prime}\right)_{\imath \jmath}=\sum_{s \varepsilon E_{\alpha \underline{1}}^{\prime}}\left(P_{m, m+\underline{1}^{\prime}-1}\right)_{\imath s}\left(\varepsilon_{m+\underline{1}^{\prime}-1, n}\right)_{s \jmath} \Rightarrow \lim \left(\varepsilon_{m, n}^{\prime}\right)_{\jmath \jmath}=0$ as $n \rightarrow \infty$.

(8) Let $\left\{I_{1}, I_{2}, \cdots, I_{f}\right\}$ be all the equivalence classes consisting of unessential selfcommunicating states. Let $I_{\beta}$ be a class with period $d(\beta)$. Partitioning it into further subclasses $\left\{I_{\beta 1}, I_{\beta 2}, \cdots, I_{\beta d(\beta)}\right\}$ as before, $\forall m \geq 1, P_{m, m+k} \mid I_{\beta k}$ is an allowable non-negative matrix. Also

$\exists M \ni n \equiv m+\left(\underline{1}^{\prime}-1\right)(\bmod d(\beta)) \Rightarrow\left(P_{m, n+M d(\beta)}\right)_{\imath \jmath} \geq \frac{1}{n+M d(\beta)}$ for $i \varepsilon I_{\beta 1}$ and $\jmath \varepsilon I_{\beta 1} ;$

So

$$
\frac{\left(P_{m, m+n M d(\beta)}\right)_{i \jmath}}{\left(P_{m, m+n M d(\beta)}\right)_{k j}} \rightarrow V^{(m)}{ }_{i h}
$$

as $n \rightarrow \infty$ for $\imath, \jmath, k \varepsilon I_{\beta d(\beta)}$ [From Hajnal]. $\forall m \geq 0 \imath, k \in I_{\beta \underline{1}}$ and $\jmath \in I_{\beta \underline{1}}, n-m=\underline{1}-\underline{1}(\bmod d(\beta))$

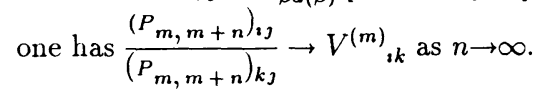

(9) Let $i$ be any state and $j \in E_{\alpha d(\alpha)}$. Let $n=r(n)(\bmod d(\alpha))$. Then $\left(P_{m, n}\right)_{\imath \jmath}=\left(P_{r(n), n}\right)_{\jmath \jmath}\left(P_{m, n}\right)_{\imath} E_{\alpha d(\alpha)}+\left(\varepsilon_{m, n}\right)_{\imath \jmath}$ where $\lim \left(\varepsilon_{m, n}\right)_{\imath \jmath}=0$ as $n \rightarrow \infty$. A similar statement holds for $j \varepsilon E_{\alpha u}, 1 \leq u \leq d(\alpha)$. To prove this, assume the opposite. Then $\exists r \ni 1 \leq r \leq d(\alpha)$ and a sequence of positive integers $\left(n_{t}\right) \ni$ if $t \geq 1$,

$$
(*) 0<\delta<\left|\left(P_{m, n t}\right)_{\imath \jmath}-\left(P_{r, n t}\right)_{\jmath \jmath}\left(P_{m, n t}\right)_{\imath E \alpha d(\alpha)}\right|
$$

where each $n_{t}=r(\bmod d(\alpha))$, and $\forall k \geq 0, P_{k, n t} \rightarrow Q_{k}, Q_{k} Q=Q_{k}, Q_{n t} \rightarrow Q=Q^{2}$. Clearly, $j$ is in a $C$-block of $Q$. (Note that $C$-blocks of $Q$ are strictly positive stochastic blocks with identical rows). If not then the $j$-th column of $Q$ is a zero column, hence a zero column of $Q_{m}$ and $Q_{r}$, and this will contradict $(*)$. Since, $\left(Q_{m}\right)_{t 1}=0$ for $\underline{1} \in T\left(=\right.$ the zero columns of $Q$ ), for $t \geq t_{0}$, $\sum_{\underline{1} \in T}\left(P_{m, n t}\right)_{\mathfrak{1} 1}<\frac{\delta}{4}$. Also, since each $\left.n_{t}=r(\bmod d(\alpha)),\left(P_{n t, n t}\right)^{\prime}\right)_{1}=0$ for $\underline{1} \notin E_{\alpha d(\alpha)} \Rightarrow Q_{\jmath \underline{1}}=0$. If $\underline{1} \notin E_{\alpha d(\alpha)} U T$, then $Q_{\underline{\jmath} \underline{1}}=0$ and therefore $Q_{\underline{1}},=0 \Rightarrow$ for $t$ large and

$$
n_{t},>>n_{t}: \sum_{\underline{1} \notin T U E_{\alpha d(\alpha)}}\left(P_{n t, n t^{\prime}}\right)_{\underline{1},}<\frac{\delta}{4} .
$$

Thus

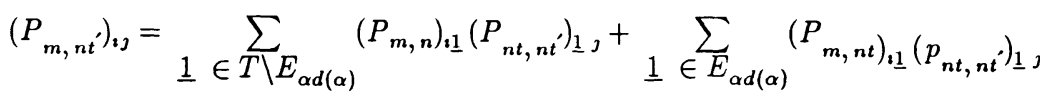

$$
\begin{aligned}
& +\sum_{\underline{1} \notin T E_{\alpha d(\alpha)}}\left(P_{m, n t}\right)_{\mathfrak{i} \underline{1}}\left(P_{n t, n t}\right)_{1},
\end{aligned}
$$

From (weak ergodicity result) (8)

$$
\left|\left(P_{m, n t^{\prime}}\right)_{i j}-\left(P_{r, n t^{\prime}}\right)_{\jmath \jmath}\left(P_{m, n t^{\prime}}\right)_{\imath E_{\alpha d(\alpha)}}\right|<\delta
$$

This is a contradiction.

\section{REFERENCES} 1. Doeblin, W., Le cas discontinu de probabilities en chaine, Publ. Fac. Sci. Univ. Masaryk
(Brno) 236 (1937), 3-13. 2. COHN, H., On a paper by Doeblin on nonhomogeneous Markov chains, Adv. Appl. Prob. 13 3. CHUNG, K.L., Markov Chains with Stationary Transition Probabilities, Springer-Verlag,
New York, 1960. 4. HAJNAL, J., On products of nonnegative matrices, Math. Proc. Cambridge Philo. Soc. 79
(1976), 521-530. 


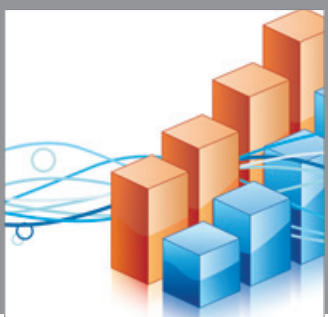

Advances in

Operations Research

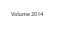

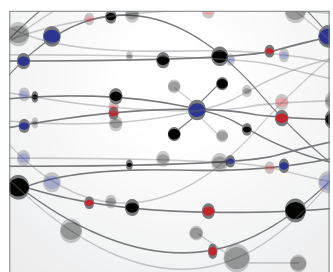

\section{The Scientific} World Journal
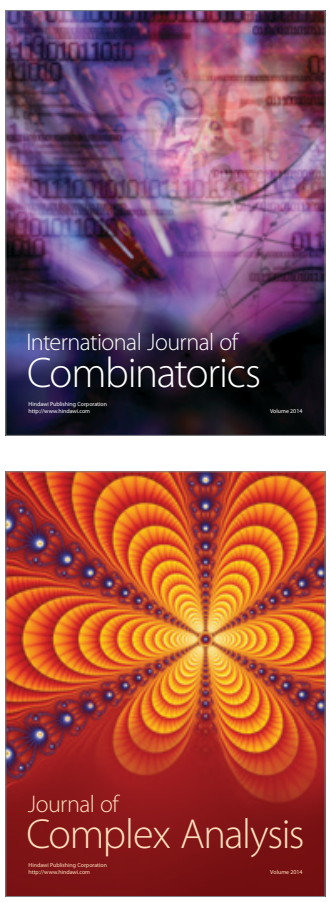

International Journal of

Mathematics and

Mathematical

Sciences
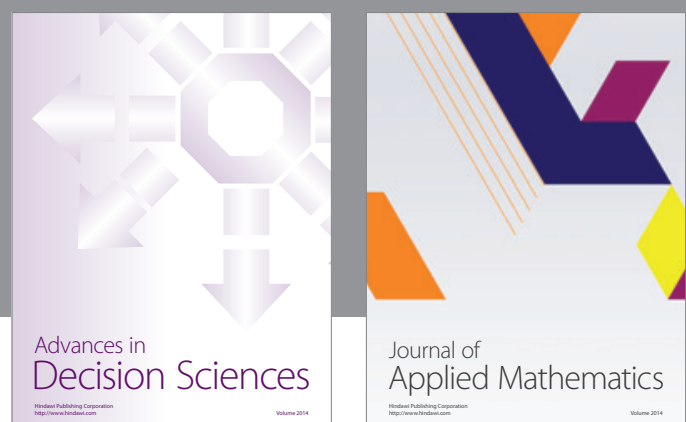

Journal of

Applied Mathematics
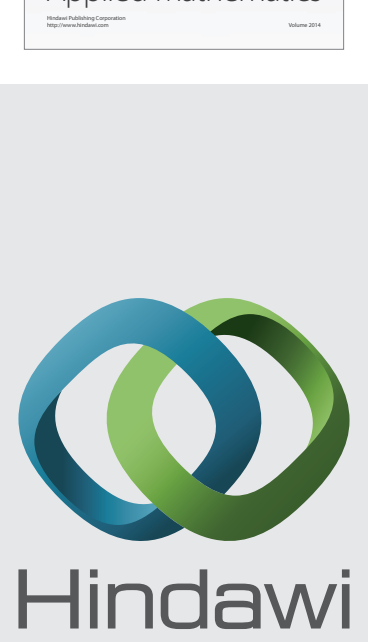

Submit your manuscripts at http://www.hindawi.com
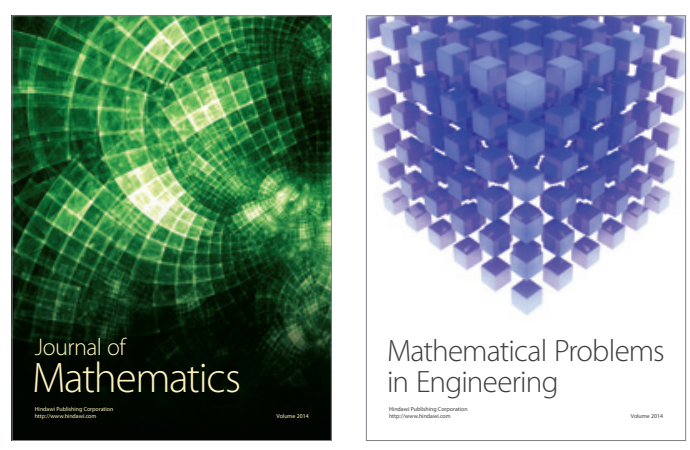

Mathematical Problems in Engineering
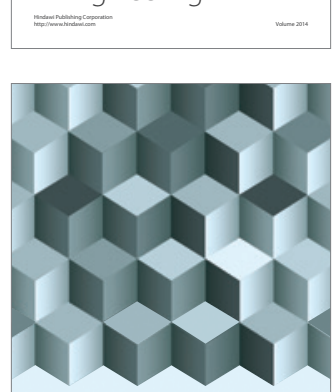

Journal of

Function Spaces
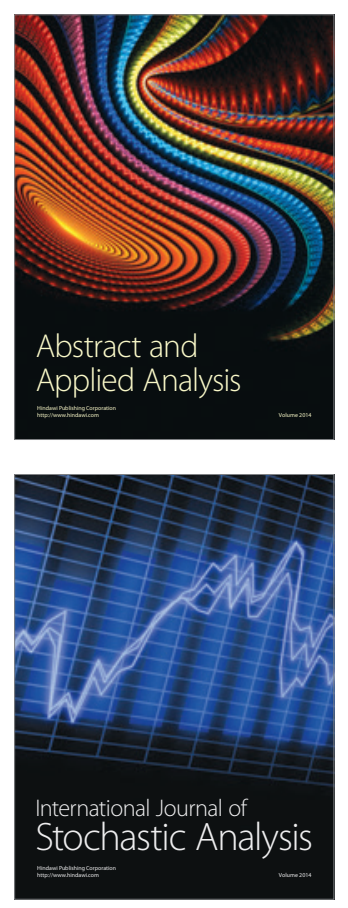

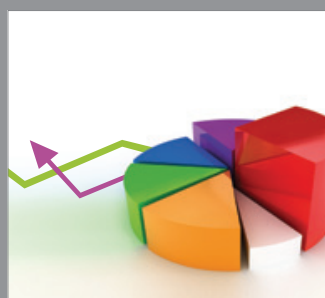

ournal of

Probability and Statistics

Promensencen
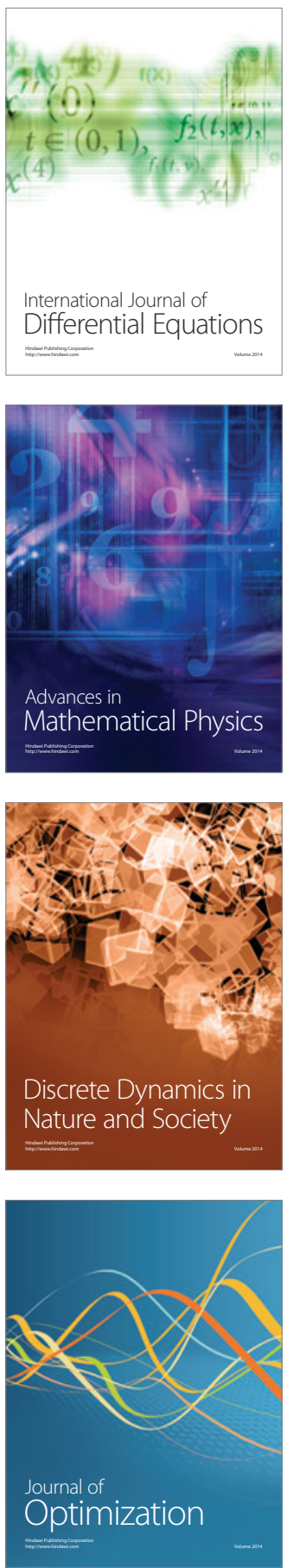